\title{
Lifestyle and neurocognition in older adults with cognitive impairments
}

\section{A randomized trial}

James A. Blumenthal, PhD, Patrick J. Smith, PhD, Stephanie Mabe, MS, Alan Hinderliter, MD, Pao-Hwa Lin, PhD, Lawrence Liao, MD, Kathleen A. Welsh-Bohmer, PhD, Jeffrey N. Browndyke, PhD, William E. Kraus, MD, P. Murali Doraiswamy, MBBS, James R. Burke, MD, PhD, and Andrew Sherwood, PhD

Neurology ${ }^{\circledR}$ 2019;92:e212-e223. doi:10.1212/WNL.0000000000006784

\section{Abstract}

\section{Objective}

To determine the independent and additive effects of aerobic exercise (AE) and the Dietary Approaches to Stop Hypertension (DASH) diet on executive functioning in adults with cognitive impairments with no dementia (CIND) and risk factors for cardiovascular disease (CVD).

\section{Methods}

A 2-by-2 factorial (exercise/no exercise and DASH diet/no DASH diet) randomized clinical trial was conducted in 160 sedentary men and women (age >55 years) with CIND and CVD risk factors. Participants were randomly assigned to 6 months of AE, DASH diet nutritional counseling, a combination of both $\mathrm{AE}$ and $\mathrm{DASH}$, or health education (HE). The primary endpoint was a prespecified composite measure of executive function; secondary outcomes included measures of language/verbal fluency, memory, and ratings on the modified Clinical Dementia Rating Scale.

\section{Results}

Participants who engaged in AE $(d=0.32, p=0.046)$ but not those who consumed the DASH $\operatorname{diet}(\mathrm{d}=0.30, p=0.059)$ demonstrated significant improvements in the executive function domain. The largest improvements were observed for participants randomized to the combined $\mathrm{AE}$ and DASH diet group $(\mathrm{d}=0.40, p=0.012)$ compared to those receiving HE. Greater aerobic fitness $(b=2.3, p=0.049)$, reduced CVD risk $(b=2.6, p=0.042)$, and reduced sodium intake $(b=0.18, p=0.024)$ were associated with improvements in executive function. There were no significant improvements in the memory or language/verbal fluency domains.

\section{Conclusions}

These preliminary findings show that $\mathrm{AE}$ promotes improved executive functioning in adults at risk for cognitive decline.

\section{ClinicalTrials.gov identifier \\ NCT01573546.}

\section{Classification of evidence}

This study provides Class I evidence that for adults with CIND, AE but not the DASH diet significantly improves executive functioning.

\author{
Correspondence \\ Dr. Blumenthal \\ James.Blumenthal@ \\ duke.edu
}

\section{MORE ONLINE}

\section{$\rightarrow$ Class of Evidence Criteria for rating therapeutic and diagnostic studies \\ NPub.org/coe}




\section{Glossary}

$\mathrm{AE}=$ aerobic exercise; $\mathrm{CDR}=$ Clinical Dementia Rating; $\mathrm{CIND}=$ cognitive impairment without dementia; COWA = Controlled Oral Word Association Test; CVD = cardiovascular disease; DASH = Dietary Approaches to Stop Hypertension; DDD = daily defined dose; ENLIGHTEN = Exercise and Nutritional Interventions for Neurocognitive Health Enhancement; FINGER = Finnish Geriatric Intervention Study to Prevent Cognitive Impairment and Disability; FSRP = Framingham Stroke Risk Profile; MoCA = Montreal Cognitive Assessment; TMT = Trail Making Test.

Cognitive impairment that does not reach sufficient severity for dementia diagnosis is associated with increased risk of progression to dementia. ${ }^{1}$ Cognitive impairment without dementia (CIND) is a term that was first used to characterize individuals whose cognitive difficulties were not sufficiently severe to meet the diagnostic criteria for dementia but were judged to be sufficient to distinguish them from healthy individuals. ${ }^{2}$ It is widely believed that known risk factors for cardiovascular disease (CVD) are also risk factors for dementia and late-life cognitive decline. ${ }^{3}$ Because there is considerable overlap in risk factors for CVD and dementia, ${ }^{4,5}$ strategies designed to reduce CVD risk also may be effective in improving neurocognition and reducing the risk of developing dementia.

Lifestyle habits, including exercise and diet, have been shown to improve CVD risk factors and are promising approaches to the prevention of neurocognitive decline. ${ }^{6}$ Observational studies have shown that higher levels of physical activity are associated with a lower risk of $\mathrm{CVD}^{7}$ and that physically active individuals are less likely to develop dementia. ${ }^{8,9}$ Intervention studies have found that aerobic exercise (AE) may improve neurocognitive functioning in healthy adults, ${ }^{10}$ although relatively few studies have included individuals with cognitive impairments. ${ }^{11}$ Dietary factors also have been shown to be associated with neurocognitive functioning. ${ }^{12}$ Observational studies have shown that variations in dietary practices and nutrient intake, including the Mediterranean $\operatorname{diet}^{13,14}$ and Dietary Approaches to Stop Hypertension (DASH) diet, ${ }^{15}$ are associated with improved cognition, although there have been few interventional studies of "whole diets." The DASH diet is part of national recommendations for the prevention and treatment of hypertension ${ }^{16}$ and has been shown reduce blood pressure, to improve other CVD risk factors, and to lower the risk of ischemic heart disease and stroke. ${ }^{17}$

\section{Methods}

\section{Eligibility and trial overview}

Exercise and Nutritional Interventions for Neurocognitive Health Enhancement (ENLIGHTEN) was a randomized clinical trial of inactive older adults with subjective memory complaints, objective evidence of cognitive impairment, and at least 1 additional CVD risk factor (besides being sedentary). Enrollment began in December 2011 and ended in March 2016. Participants completed a comprehensive battery of neurobehavioral tests, along with assessments of dietary habits, cardiorespiratory fitness, and CVD biomarkers. After completion of baseline assessments, a 2 (AE)-by-2 (DASH diet) factorial design was used with participants randomly assigned, in roughly equal numbers, to 1 of 4 groups: $\mathrm{AE}$ alone (AE), DASH diet alone (DASH), a combination of AE and the DASH diet (AE + DASH), or health education (HE). At the conclusion of the 6-month intervention period, measurements of neurocognition, dietary consumption, cardiorespiratory fitness, and CVD risk factors were repeated. Assessors were blinded to patients' treatment group assignment.

\section{Standard protocol approvals, registrations, and patient consents}

This study was approved by the Institutional Review Board at Duke University Medical Center, and written informed consent was obtained from all participants. The trial was registered at ClinicalTrials.gov (NCT01573546).

\section{Participants}

One hundred sixty older adults were recruited from newspaper and television advertisements, physician referrals, and patient mailings through recruitment databases at the Duke Aging Center and the Duke Alzheimer's Disease Research Center. Inclusion criteria included adults $\geq 55$ years of age with subjective cognitive complaints as reflected by a score of $\geq 0.5$ on the Mail-in Cognitive Function Screening Instrument and a score of 19 to 25 on the Montreal Cognitive Assessment (MoCA) or a score of $\leq 12$ on letter fluency or $\leq 15$ on animal fluency. Participants also had to be sedentary (i.e., potential participants did not engage in physical activities intentionally designed to increase their heart rates for the purpose of improving their physical fitness more than twice a week and for $<30$ minutes) and have either documented CVD or at least 1 additional CVD risk factor. Patients with dementia or major psychiatric illness (e.g., schizophrenia, acute suicidality, psychosis) and those who were unable to exercise due to musculoskeletal problems or cardiac abnormalities (e.g., class IV heart failure, unstable angina) were excluded.

\section{Stratification and randomization}

A conditional randomization procedure was used to stratify participants according to sex (male/female), age (55-69/ $\geq 70$ years), MoCA score $(19-23 / \geq 24)$, and history of CVD $(+/-)$. To maintain allocation concealment, group assignments were placed in sealed envelopes that were opened at the time of randomization. Research staff engaged in randomization and assessments were blinded to participants' group assignment. All assessments were performed at Duke University Medical Center. 


\section{Outcome measures}

The primary endpoint was a global measure of executive cognitive functioning. On the basis of evidence that improvements in neurocognition are specific to enhancements in executive-control processes, ${ }^{18}$ we hypothesized that the interventions would especially improve executive function. These processes and the brain areas that support them are believed to be differentially affected by aging and improved with exercise training $^{19,20}$ (Class of Evidence: I). Secondary outcomes included global measures of memory, language/verbal fluency, and Clinical Dementia Rating (CDR).

\section{Assessment procedures}

\section{Assessment of neurocognitive functioning}

Neurocognitive functioning was assessed with a modified test battery recommended by the Neuropsychological Working Group for vascular cognitive disorders ${ }^{21}$ designed to tap, a priori, 3 key domains of cognitive functioning. Our primary outcome was executive function, assessed by the Trail Making Test (TMT), the Stroop Test, the Digit Span Forward and Backward subtest from the Wechsler Adult Intelligence Scale, the Digit Symbol Substitution Test from the Wechsler Adult Intelligence Scale, the Ruff 2 \& 7 Test, and Animal Naming. Secondary domains included memory, assessed by the Hopkins Verbal Learning Test-Revised and the Medical College of Georgia Complex Figure Test, and language/verbal fluency, assessed by the Controlled Oral Word Association Test (COWA) and the Animal Naming Test, which also was included as part of the global executive function measure.

In addition, to examine changes in global cognitive function, we used the modified CDR Sum of Boxes to characterize 6 domains of cognitive and functional performance. ${ }^{22}$ This clinical interview was administered and scored by a trained rater who was blinded to patients' intervention group status.

\section{Aerobic capacity and physical activity}

\section{Exercise testing}

Participants underwent a maximal graded exercise treadmill test using the Duke-Wake Forest protocol in which workloads were increased at a rate of 1 metabolic equivalent per minute. ${ }^{23}$ Expired air was collected by mouthpiece for quantification of minute ventilation, oxygen consumption, and carbon dioxide production with the Parvo Medics TrueOne measurement system (model 2400; Parvo Medics, Sandy, UT).

\section{Accelerometry}

Physical activity during daily life was quantified with the Kenz Lifecorder Plus accelerometer (model NL-2160; Suzuken Co. Ltd, Nagoya, Japan). Patients wore the accelerometer for 48 consecutive hours to obtain an average number of daily steps.

\section{Dietary habits}

Diet was assessed by the Block Food Frequency Questionnaire and a 4-day food diary. We sought to quantify the degree of adherence of participants' daily dietary intake of the DASH diet. To quantify the DASH eating pattern, we used a modified DASH scoring algorithm adopted from Epstein et al. ${ }^{24}$ and Folsom and colleagues. ${ }^{25}$ Dietary intake from a 4-day diary was used for all dietary components for which a score could be derived (fruits, vegetables, dairy, grains, fat calories, saturated fat calories, and sodium), with only those categories that could not be quantified by the diary being generated from the Block Food Frequency Questionnaire (meats, nuts/seeds/legumes, and sweets).

\section{CVD risk}

A global measure of CVD risk using a composite rank procedure was used to quantify CVD risk. Blood pressure was determined according to Seventh Report of the Joint National Committee on Prevention, Detection, Evaluation, and Treatment of High Blood Pressure guidelines. ${ }^{16}$ Glucose and lipids were obtained from fasting blood samples drawn between 8 and 9 AM; assays were measured enzymatically (LabCorp Inc, Burlington, NC). CVD medications were documented by chart review and confirmed by examination of patient pill bottles to determine daily defined dose (DDD) of CVD medications. ${ }^{26}$

The Framingham Stroke Risk Profile (FSRP) was used to quantify baseline CVD risk before randomization. ${ }^{27}$ Because several determinants of the FSRP score are nonmodifiable (e.g., age, history of CVD, atrial fibrillation, ECG-determined left ventricular hypertrophy, history of treated hypertension), the FSRP was used as a control variable, not a postintervention endpoint.

\section{APOE \& 4 genotyping}

$A P O E \& 4$ allele genotyping was completed with the TaqMan SNP Genotyping Assay (Life Technologies, Grand Island, NY).

\section{Interventions}

\section{Aerobic exercise}

Participants performed AE for 6 months. For the initial 3 months, participants exercised 3 times a week at a level of $70 \%$ to $85 \%$ of their initial peak heart rate reserve under supervision at a cardiac rehabilitation facility in central North Carolina. $\mathrm{AE}$ consisted of 10 minutes of warm-up exercises followed by 35 minutes of continuous walking or (stationary) cycling. During the subsequent 3 months, participants exercised 3 times per week on their own at home at $70 \%$ to $85 \%$ heart rate reserve, which they documented in weekly exercise logs that were reviewed weekly by study personnel. Participants in the AE alone condition did not receive any counseling on the DASH diet and were encouraged to follow their usual diets.

\section{DASH diet}

Participants in the DASH eating plan condition received instruction on modifying the content of their diet to meet DASH guidelines. Education on the DASH diet and feedback on participants' adherence were provided by a nutritionist in a series of half-hour sessions conducted weekly for the initial 12 weeks and then biweekly for weeks 13 to 24 . Participants in the DASH-alone condition were asked not to exercise. 


\section{AE + DASH}

Participants in the AE + DASH condition received both the $\mathrm{AE}$ and $\mathrm{DASH}$ interventions as described above.

\section{Health education control group}

The HE control group received weekly 30-minute educational phone calls for 3 months and then biweekly for 3 months. Phone calls were conducted by a health educator (a physician's assistant) on relevant CVD health-related topics. Participants were asked to maintain their usual dietary and exercise habits for 6 months until they were reevaluated.

\section{Data analysis}

All analyses of treatment-related changes were conducted using general linear models with SAS 9.4 (PROC GLM; SAS Institute, Cary, NC). Consistent with a 2-by-2 factorial design, treatment improvements were first tested contrasting 2 a priori factors: an exercise factor (AE + DASH and $\mathrm{AE})$ vs noexercise (DASH diet alone and HE) and a DASH factor (AE + DASH and DASH alone) vs no-DASH (AE and HE).

Neurocognitive function was assessed in 3 domains. Our primary prespecified outcome was global executive function (TMT-A, TMT-B, TMT B-A, Ruff 2 \& 7 [automatic and controlled processing speed], Stroop [word, color, colorword, and interference], Digit Symbol Substitution Test, Digit Span [Forward and Backward], and Animal Naming). Secondary cognitive outcomes included assessments of memory (Hopkins Verbal Learning Test [total learning and retention] and Medical College of Georgia [short and long delay]), and language/verbal fluency (Animal Naming and COWA). For analyses of treatment-related changes in neurocognition, separate rank-based composites were created, combining results from all neurocognitive subtests within each cognitive domain before and after treatment. This method had been advocated for increasing power while simultaneously lowering type I error rates. ${ }^{28,29}$ A mean rank was then created by averaging across all subtests for the executive function, memory, and language/verbal fluency domains at each time point. If the global outcome was significant, a closed testing procedure was performed to determine which subtests were affected by the intervention as an explanatory follow-up test. ${ }^{30}$ Post hoc comparisons were performed to examine the potential additive effect of combining exercise with the DASH diet on neurocognition. To characterize the magnitude of intervention-related changes, improvements in the composite measures of neurocognition were reported with the Cohen $\mathrm{d}$, an established index of effect size, as well as estimating predicted age on the basis of neurocognitive performance. ${ }^{31}$ In addition, for analyses of neurocognitive outcomes, we controlled a priori for education, FSRP, APOE genotype, and baseline MoCA score, as well as CVD medication burden and long-term use of anti-inflammatory medications. We estimated that, for a given main effect, our sample size would yield a power of 0.80 to detect a treatment effect of $\approx 0.48 \mathrm{SD}$.
Analyses of changes in fitness, dietary composition, and CVD risk were conducted with general linear models with a priori covariates controlling for stratification variables (age, sex, CVD history, and MoCA score), race, and the baseline level of each outcome. To minimize the number of statistical tests, analyses of CVD risk factors also used a composite measure of all nonoverlapping CVD outcomes (systolic and diastolic blood pressures, low-density lipoprotein cholesterol, highdensity lipoprotein cholesterol, very low-density lipoprotein cholesterol, DDD of CVD medications, body mass index, insulin, glucose, and triglycerides). Analyses of treatment effects followed intention to treat with any missing data managed by Markov chain Monte Carlo multiple imputation methods available in SAS (PROC MI) and 100 imputations. To explore which elements of the exercise and DASH diet interventions may have contributed to changes in neurocognition, we also examined the associations between changes in fitness, components of the DASH diet, and CVD risk factors. To minimize the number of possible tests, we examined a priori improvements in peak $\mathrm{VO}_{2}$, select DASH diet components (magnesium, potassium, sodium, and calcium), and our composite marker of CVD risk in relation to neurocognition, controlling for the same variables as the primary analyses of neurocognitive change.

\section{Data availability}

Anonymized data published within this article will be shared by request from any qualified investigator 3 years from the date of publication of this article.

\section{Results}

\section{Participant flow}

Of 431 inquires, 271 participants were screened out, leaving 160 participants who were enrolled and randomized as follows: to DASH alone $(\mathrm{n}=41), \mathrm{AE}$ alone $(\mathrm{n}=41), \mathrm{AE}+$ DASH $(n=40)$, and HE $(n=38)$ (figure 1$)$. The primary reasons for exclusion were lack of objective evidence of cognitive impairment (i.e., MoCA score $\geq 25$ ), medical contraindications (e.g., class III or IV heart failure, recent cancer treatment, unable to walk without assistance), and logistical barriers (e.g., lives too far from an exercise facility, unable to commit to 6 months of study participation).

\section{Background characteristics}

Background and clinical characteristics for the sample are shown in table 1. Participants tended to be older (mean age 65.4 [SD 6.8] years) and female (66\%) and were evenly divided between whites and minorities. Twenty-one (13\%) participants had documented coronary heart disease (i.e., myocardial infarction or revascularization procedure), and 7 additional participants (4\%) had a history of a TIA without residual deficits. Participants exhibited an average atherosclerotic CVD risk score of 15.4 (SD 11.2) and an FSRP score of 9.7 (SD 4.2), suggesting moderate risk of CVD events. Examination of individual CVD risk factors revealed that hypertension (76\%) and hyperlipidemia (48\%) were 


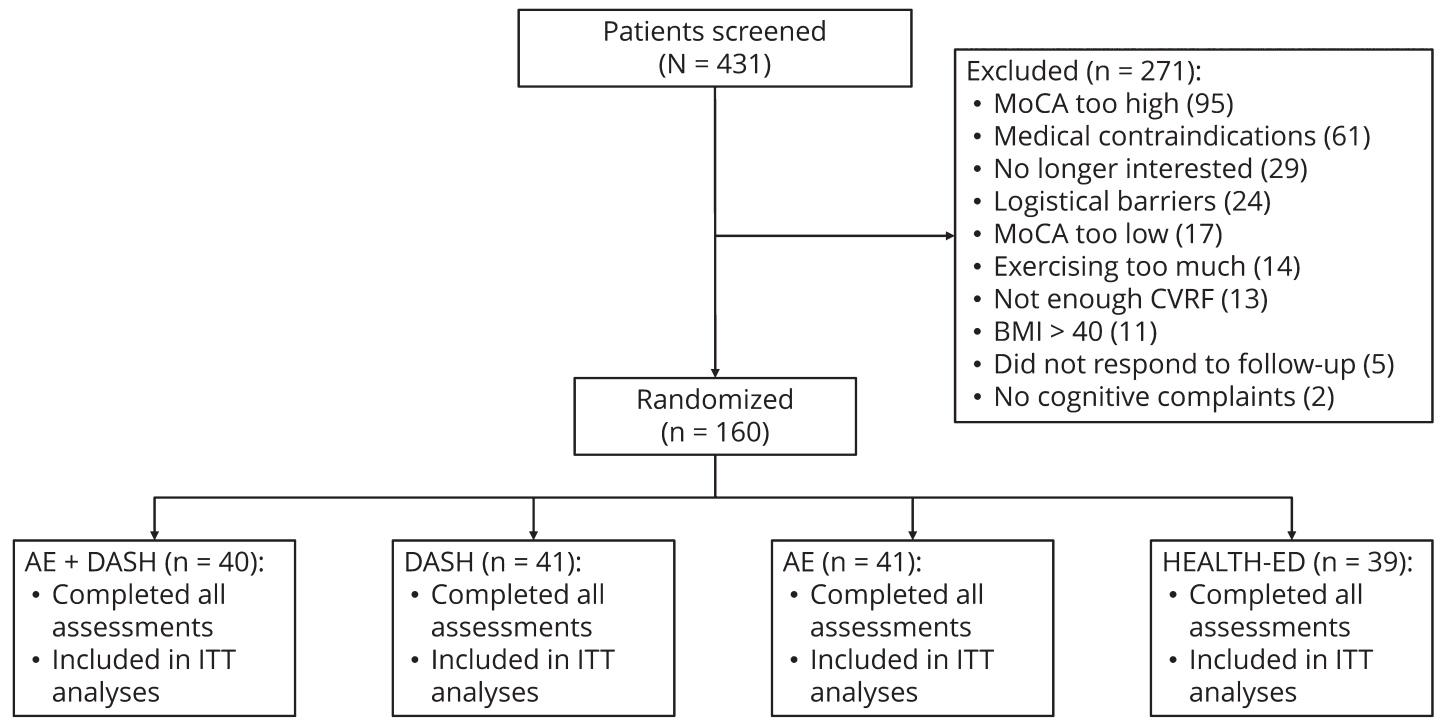

Twenty-four patients were excluded for logistic reasons (e.g., they had no transportation, lived too far from Duke University Medical Center, or had plans for extended absences during the 6 months of active intervention). These patients were excluded before completing baseline assessments and before randomization. $\mathrm{AE}=$ aerobic exercise; $\mathrm{BMI}$ = body mass index; $\mathrm{CVRF}=$ cardiovascular risk factor; DASH = Dietary Approaches to Stop Hypertension; ITT = intention to treat; MoCA = Montreal Cognitive Assessment.

most common. Among the 144 participants who provided valid APOE data, 47 (33\%) had at least 1 APOE $\varepsilon 4$ allele.

\section{Adherence to the interventions}

The AE + DASH and AE alone groups attended an average of 31.5 (SD 4.1) sessions of a target of 36 supervised exercise sessions (88.6\%) and 32.5 (SD 9.7) of the required 36 homebased sessions (90.4\%). We note that most patients attended at least $75 \%$ of the supervised exercise sessions, with 71 participants $(88 \%)$ attending at least $75 \%$ of supervised sessions and 67 participants (83\%) attending at least $75 \%$ of homebased sessions. DASH counseling participants in the AE + DASH and DASH-only groups attended an average of 17.9 (SD 0.5) of 18 scheduled dietary classes (99.6\%). Participants in the HE condition participated in an average of 17.5 (SD 1.7) of 18 telephone sessions (97\%), lasting an average of 12.2 minutes (SD 3.2 minutes).

\section{Intervention manipulation checks}

\section{Changes in aerobic fitness and physical activity}

Participants in the exercise conditions exhibited greater improvements in aerobic fitness, treadmill time, 6-minute walk distance, and daily physical activity (table 2 ). Compared with participants in the nonexercise conditions, exercisers achieved greater improvements in aerobic capacity $\left(\mathrm{VO}_{2}\right.$ peak $\mathrm{M}=1.7$ [1.2-2.1] mL $/ \mathrm{kg} / \mathrm{min}$ vs $\mathrm{M}=-0.3[-0.8$ to 0.1$] \mathrm{mL} / \mathrm{kg} /$ minute, $p<0.001)$, corresponding to nearly a $10 \%$ differential improvement $(9.6 \%[6.7 \%-12.4 \%]$ vs $-0.2 \%$ [ $-3.1 \%$ to $2.7 \%])$. Greater improvements in other fitness indexes also were observed in the exercise groups compared to nonexercisers, including greater treadmill duration $(p<0.001)$, 6-minute walk distance $(p<$ $0.001)$, and daily steps $(p<0.001)$.

\section{Changes in DASH diet and nutritional composition}

Participants in the DASH diet conditions exhibited greater improvements in DASH eating plan compared with participants in the non-DASH conditions $(2.1[1.8-2.4]$ vs 0.0 [-0.3 to 0.3$], p<0.001$ ) (table 2 ), corresponding to improvements of 1.9 (1.4-2.3) units in AE + DASH, 2.3 (1.9-2.7) in DASH alone, -0.1 ( -0.5 to 0.3) in AE alone, and 0.1 ( -0.3 to 0.6$)$ in HE (table 2). Examination of individual DASH diet components revealed that participants in the DASH diet conditions demonstrated increased consumption of potassium $(p<0.001)$, magnesium $(p<0.001)$, and calcium $(p<0.001)$ and a decrease in dietary sodium $(p<$ 0.001 ), whereas non-DASH participants consumed fewer DASH diet nutrients.

\section{Changes in CVD risk factors}

Examination of our composite CVD risk measure demonstrated that participants in both the DASH $(\mathrm{d}=0.33, p=$ $0.040)$ and exercise $(\mathrm{d}=0.32, p=0.048)$ factors showed improvements in CVD risk factors. Follow-up examination of specific CVD risk factors revealed that participants in the DASH diet conditions demonstrated a reduction in total cholesterol $(-10.5[5.6-15.5] \mathrm{mg} / \mathrm{dL}, p=0.015)$, reduced weight $(-4.0[2.5-5.5] \mathrm{lb}, p=0.018)$, reduced low-density lipoprotein cholesterol $(-7.1[3.0-11.3] \mathrm{mg} / \mathrm{dL}, p=0.045)$, and a reduction in the number of antihypertensive medications taken ( -0.24 [ -0.06 to -0.42$] \mathrm{DDD}, p=0.003)$. There were no significant intervention group differences in systolic blood pressure ( $p=0.189$ for DASH factor, $p=0.806$ for exercise factor). However, the DASH factor groups (AE + DASH and DASH alone) demonstrated reductions in blood pressure medications, decreasing their total blood pressure 
Table 1 Background and demographic characteristics of the sample by intervention group: AE + DASH, AE alone, DASH diet alone, and $\mathrm{HE}$

\begin{tabular}{|c|c|c|c|c|c|c|}
\hline \multirow[b]{2}{*}{ Variable } & \multicolumn{2}{|l|}{ Exercise } & \multicolumn{2}{|l|}{ No exercise } & \multirow[b]{2}{*}{$\begin{array}{l}\text { Total } \\
\text { cohort } \\
(n=160)\end{array}$} & \multirow[b]{2}{*}{$\begin{array}{l}p \text { Value for } \\
\text { overall group } \\
\text { differences }\end{array}$} \\
\hline & $\begin{array}{l}\text { DASH } \\
(A E+D A S H) \\
(n=40)\end{array}$ & $\begin{array}{l}\text { No-DASH } \\
(A E) \\
(n=41)\end{array}$ & $\begin{array}{l}\text { DASH } \\
\text { (DASH) } \\
(n=41)\end{array}$ & $\begin{array}{l}\text { No-DASH } \\
(\mathrm{HE}) \\
(\mathrm{n}=38)\end{array}$ & & \\
\hline Age, y & $64.9(6.2)$ & $65.8(7.3)$ & $66.0(7.1)$ & $64.7(6.6)$ & $65.4(6.8)$ & 0.737 \\
\hline Male sex, n (\%) & $14(35)$ & $12(31)$ & $15(36)$ & $12(32)$ & $54(34)$ & 0.954 \\
\hline \multicolumn{7}{|l|}{ Ethnicity, n (\%) } \\
\hline White & $18(45)$ & $18(46)$ & $25(61)$ & $22(58)$ & $85(53)$ & 0.237 \\
\hline Black & $22(55)$ & $20(51)$ & $16(39)$ & $16(42)$ & $74(46)$ & \\
\hline Other & $0(0)$ & $1(3)$ & $0(0)$ & $(0)$ & $1(1)$ & \\
\hline \multicolumn{7}{|l|}{ Married, n (\%) } \\
\hline Married & $22(55)$ & $20(51)$ & $24(59)$ & $18(47)$ & $86(54)$ & 0.794 \\
\hline Divorced/widowed & $16(40)$ & $17(44)$ & $13(32)$ & $17(45)$ & $63(39)$ & \\
\hline Single, never married & $2(5)$ & $2(5)$ & $4(10)$ & $3(8)$ & $11(7)$ & \\
\hline \multicolumn{7}{|l|}{ Level of education, n (\%) } \\
\hline Less than high school & $0(0)$ & $0(0)$ & $0(0)$ & $1(3)$ & $1(1)$ & 0.386 \\
\hline High school graduate & $3(8)$ & $3(8)$ & $4(10)$ & $2(5)$ & $14(9)$ & \\
\hline Some college & $13(33)$ & $14(36)$ & $10(24)$ & $11(29)$ & $48(30)$ & \\
\hline College degree/graduate school & $24(61)$ & $22(55)$ & $27(66)$ & $24(63)$ & $97(60)$ & \\
\hline \multicolumn{7}{|l|}{ Employment status, n (\%) } \\
\hline Full-time & $12(30)$ & $9(23)$ & $9(22)$ & $8(21)$ & $38(24)$ & 0.888 \\
\hline Part-time & $6(15)$ & $3(8)$ & $6(15)$ & $6(16)$ & $21(13)$ & \\
\hline Retired & $16(40)$ & $22(56)$ & $22(54)$ & $20(53)$ & $82(51)$ & \\
\hline Not employed & $6(15)$ & $5(13)$ & $4(10)$ & $4(11)$ & $19(12)$ & \\
\hline MoCA score & $25.0(2.5)$ & $24.8(2.7)$ & $24.4(2.7)$ & $24.4(2.3)$ & $24.7(2.6)$ & 0.470 \\
\hline MICFSI score & $5.1(3.0)$ & $4.7(2.8)$ & $4.5(2.1)$ & $4.8(2.3)$ & $4.8(2.6)$ & 0.756 \\
\hline Phonemic fluency & $12.2(3.8)$ & $11.2(3.6)$ & $11.6(3.1)$ & $12.7(3.6)$ & $12.8(4.0)$ & 0.262 \\
\hline \multicolumn{7}{|l|}{ Clinical characteristics, n (\%) } \\
\hline Blood pressure medications & $27(68)$ & $30(77)$ & $32(78)$ & $32(84)$ & $122(76)$ & 0.376 \\
\hline Cholesterol medications & $17(43)$ & $15(38)$ & $23(56)$ & $21(55)$ & $77(48)$ & 0.222 \\
\hline Diabetes medications & $6(15)$ & $8(21)$ & $7(17)$ & $6(16)$ & $27(17)$ & 0.953 \\
\hline Obstructive sleep apnea & $9(23)$ & $9(23)$ & $8(20)$ & $13(34)$ & $40(25)$ & 0.472 \\
\hline \multicolumn{7}{|l|}{ Cardiovascular risk factors } \\
\hline Hypertension, n (\%) & $27(71)$ & $29(74)$ & $31(78)$ & $31(84)$ & $123(77)$ & 0.594 \\
\hline Systolic blood pressure, mm Hg & $130(14.7)$ & $131.5(13.4)$ & $128.1(14.2)$ & $131.7(12.7)$ & $130.3(13.7)$ & 0.632 \\
\hline Diastolic blood pressure, $\mathrm{mm} \mathrm{Hg}$ & $76.7(6.5)$ & $75.7(7.0)$ & $76.5(7.1)$ & $77.1(8.8)$ & $76.0(7.4)$ & 0.393 \\
\hline Hyperlipidemia, n (\%) & $25(66)$ & $21(54)$ & $25(63)$ & $22(59)$ & $97(61)$ & 0.879 \\
\hline Total cholesterol, mg/dL & $199.6(44.1)$ & $192.3(36.6)$ & $185.9(38.9)$ & $195.6(28.3)$ & $193.2(38.7)$ & 0.607 \\
\hline High-density lipoprotein, mg/dL & $56.0(14.2)$ & $60.1(16.3)$ & $58.8(17.2)$ & $59.4(20.5)$ & $58.5(17.0)$ & 0.758 \\
\hline Low-density lipoprotein, mg/dL & $117.9(38.3)$ & $109.4(31.6)$ & $104.7(37.0)$ & $113.5(27.0)$ & $111.3(34.5)$ & 0.398 \\
\hline
\end{tabular}


Table 1 Background and demographic characteristics of the sample by intervention group: AE + DASH, AE alone, DASH diet alone, and HE (continued)

\begin{tabular}{|c|c|c|c|c|c|c|}
\hline \multirow[b]{2}{*}{ Variable } & \multicolumn{2}{|l|}{ Exercise } & \multicolumn{2}{|l|}{ No exercise } & \multirow[b]{2}{*}{$\begin{array}{l}\text { Total } \\
\text { cohort } \\
(n=160)\end{array}$} & \multirow[b]{2}{*}{$\begin{array}{l}p \text { Value for } \\
\text { overall group } \\
\text { differences }\end{array}$} \\
\hline & $\begin{array}{l}\text { DASH } \\
(A E+D A S H) \\
(n=40)\end{array}$ & $\begin{array}{l}\text { No-DASH } \\
(\mathrm{AE}) \\
(\mathrm{n}=41)\end{array}$ & $\begin{array}{l}\text { DASH } \\
\text { (DASH) } \\
(n=41)\end{array}$ & $\begin{array}{l}\text { No-DASH } \\
(\mathrm{HE}) \\
(\mathrm{n}=38)\end{array}$ & & \\
\hline Very low-density lipoprotein, mg/dL & $25.7(11.4)$ & $22.8(10.1)$ & $22.4(7.9)$ & $22.7(9.9)$ & $23.0(9.8)$ & 0.345 \\
\hline Diabetes mellitus, n (\%) & $8(20)$ & $11(28)$ & $9(22)$ & $7(18)$ & $37(23)$ & 0.841 \\
\hline Glucose, $\mathrm{mmol} / \mathrm{L}$ & $98.9(21.7)$ & $101.7(19.4)$ & $104.4(38.9)$ & $102.4(18.3)$ & $101.9(25.9)$ & 0.800 \\
\hline Insulin, ulU/mL & $16.3(10.0)$ & $13.1(6.9)$ & $12.2(5.9)$ & $13.3(8.3)$ & $13.8(8.0)$ & 0.130 \\
\hline Triglycerides, mg/dL & $98.9(21.7)$ & $102.0(19.5)$ & $104.4(38.8)$ & $102.4(18.3)$ & $117.1(49.3)$ & 0.314 \\
\hline Obesity, n (\%) & $30(79)$ & $22(56)$ & $24(60)$ & $23(62)$ & $104(65)$ & 0.264 \\
\hline Body mass index, $\mathrm{kg} / \mathrm{m}^{2}$ & $33.3(3.7)$ & $31.6(5.6)$ & $32.5(4.6)$ & $32.7(5.1)$ & $32.5(4.8)$ & 0.808 \\
\hline Current smoker, n (\%) & $3(8)$ & $1(3)$ & $0(0)$ & $2(3)$ & $6(4)$ & 0.309 \\
\hline Pack-years, median (IQR) & $2.3(14.5)$ & $1.5(10.0)$ & $0(5.0)$ & $1.8(15.0)$ & $0.8(10.3)$ & 0.370 \\
\hline $\begin{array}{l}\text { Long-term anti-inflammatory medications, } \\
\mathrm{n}(\%)\end{array}$ & $8(5)$ & $2(1)$ & $3(2)$ & $2(1)$ & $15(9)$ & 0.064 \\
\hline Total CVD medications, DDD & $1.9(1.8)$ & $2.3(2.0)$ & $2.8(2.1)$ & $3.2(2.5)$ & $2.5(2.1)$ & 0.054 \\
\hline Blood pressure medications, DDD & $1.4(1.5)$ & $1.7(2.0)$ & $1.6(1.4)$ & $2.1(2.1)$ & $1.7(1.8)$ & 0.314 \\
\hline Cholesterol medications, DDD & $0.4(0.6)$ & $0.5(0.7)$ & $1.1(1.5)$ & $0.9(1.2)$ & $0.7(1.1)$ & 0.012 \\
\hline Diabetes medications, DDD & $0.1(0.4)$ & $0.2(0.5)$ & $0.1(0.3)$ & $0.2(0.5)$ & $0.2(0.4)$ & 0.813 \\
\hline History of CVD, $\mathrm{n}(\%)$ & $7(18)$ & $6(15)$ & $8(20)$ & $4(11)$ & $27(17)$ & 0.786 \\
\hline Family history of CVD, $\mathrm{n}(\%)$ & $11(29)$ & $13(33)$ & $14(35)$ & $12(32)$ & $50(31)$ & 0.913 \\
\hline Family history of dementia, $n(\%)$ & $5(13)$ & $7(17)$ & $3(7)$ & $7(18)$ & $22(14)$ & 0.368 \\
\hline APOE $\varepsilon 4$ allele $(\geq 1), n(\%)$ & $11(29)$ & $10(29)$ & $8(20)$ & $19(50)$ & $48(32)$ & 0.058 \\
\hline CVD risk factors, $\mathrm{n}$ & $2.8(1.3)$ & $2.6(1.1)$ & $2.7(1.2)$ & $2.6(1.1)$ & $2.8(1.1)$ & 0.790 \\
\hline Atherosclerotic CVD risk profile & $14.3(9.3)$ & $16.1(10.1)$ & $15.3(12.0)$ & $14.6(12.7)$ & $15.4(11.2)$ & 0.943 \\
\hline FSRP & $8.6(3.7)$ & $9.0(3.1)$ & $8.6(4.1)$ & $8.6(3.6)$ & $8.6(3.6)$ & 0.836 \\
\hline Charlson Comorbidity Index & $0.8(1.1)$ & $0.8(1.2)$ & $0.8(1.4)$ & $0.6(0.8)$ & $0.8(1.1)$ & 0.902 \\
\hline \multicolumn{7}{|l|}{ Aerobic fitness/physical activity/diet indexes } \\
\hline Peak $\mathrm{Vo}_{2}, \mathrm{~mL} / \mathrm{kg} / \mathrm{min}$ & $18.3(4.4)$ & $18.4(4.7)$ & $18.7(4.1)$ & $18.1(4.8)$ & $18.3(4.5)$ & 0.938 \\
\hline Exercise treadmill duration, $\min$ & $7.6(1.9)$ & $8.0(2.1)$ & $8.0(1.9)$ & $7.6(2.3)$ & $7.8(2.1)$ & 0.819 \\
\hline 6-min walk distance, $\mathrm{m}$ & $464.5(51.1)$ & $463.1(66.8)$ & $459.2(52.5)$ & $462.3(54.2)$ & $461.8(56.4)$ & 0.981 \\
\hline Accelerometer light activity & $51.5(27.1)$ & $50.5(21.9)$ & $53.1(24.8)$ & $48.3(27.9)$ & $50.9(25.4)$ & 0.865 \\
\hline Moderate activity & $8.4(8.4)$ & $7.3(9.7)$ & $8.2(8.8)$ & $8.5(9.1)$ & $8.1(8.9)$ & 0.936 \\
\hline Vigorous activity & $0.1(0.2)$ & $0.1(0.3)$ & $0.2(0.4)$ & $0.1(0.3)$ & $0.1(0.3)$ & 0.895 \\
\hline Accelerometer total steps, $n$ & $5,325(2,545)$ & $5,120(2074)$ & $5,558(2,696)$ & $5,083(3,012)$ & $5,272(25)$ & 0.839 \\
\hline DASH diet score & $3.4(1.0)$ & $3.4(1.2)$ & $3.6(1.0)$ & $3.7(1.3)$ & $3.5(1.1)$ & 0.481 \\
\hline
\end{tabular}

Abbreviations: AE = aerobic exercise; CVD = cardiovascular disease; DASH = Dietary Approaches to Stop Hypertension; DDD = daily defined dose; FSRP = Framingham stroke risk profile; $\mathrm{HE}$ = health education; IQR = interquartile range; MICFSI = Mail-in Cognitive Function Screening Instrument.

$p$ Values uncorrected for baseline treatment group differences. 
Table 2 Postintervention values adjusted for preintervention values of each respective variable, along with age, sex, race, history of CVD event, and pretreatment MoCA score

\begin{tabular}{|c|c|c|c|c|c|c|c|}
\hline \multirow[b]{3}{*}{ Variable } & \multicolumn{4}{|c|}{ Individual intervention groups (by exercise factor) } & \multirow[b]{3}{*}{$\begin{array}{l}\text { Exercise } \\
\text { factor }\end{array}$} & \multirow[b]{3}{*}{$\begin{array}{l}\text { DASH } \\
\text { factor }\end{array}$} & \multirow[b]{3}{*}{$\begin{array}{l}\text { Combc } \\
\text { vs HE }\end{array}$} \\
\hline & \multicolumn{2}{|l|}{ Exercise } & \multicolumn{2}{|l|}{ No exercise } & & & \\
\hline & $\begin{array}{l}\text { DASH } \\
(A E+D A S H) \\
(n=40)\end{array}$ & $\begin{array}{l}\text { No-DASH } \\
\text { (AE) }(n=41)\end{array}$ & $\begin{array}{l}\text { DASH } \\
\text { (DASH) } \\
(n=41)\end{array}$ & $\begin{array}{l}\text { No-DASH } \\
\text { (HE) }(n=38)\end{array}$ & & & \\
\hline \multicolumn{8}{|c|}{ Fitness and physical activity outcomes } \\
\hline Peak $\mathrm{Vo}_{2}, \mathrm{~mL} / \mathrm{kg} / \mathrm{min}^{\mathrm{a}, \mathrm{b}}$ & $20.4(19.8-21.1)$ & $\begin{array}{l}19.6 \\
(19.0-20.2)\end{array}$ & $\begin{array}{l}18.1 \\
(17.5-18.7)\end{array}$ & $\begin{array}{l}17.9 \\
(17.3-18.6)\end{array}$ & $<0.001$ & 0.182 & $<0.001$ \\
\hline ETT time, mins ${ }^{b}$ & $10.0(9.5-10.4)$ & $9.3(8.9-9.7)$ & $7.7(7.3-8.1)$ & $7.5(7.1-8.0)$ & $<0.001$ & 0.064 & $<0.001$ \\
\hline Total steps, $\mathbf{n}^{\mathbf{b}}$ & $\begin{array}{l}6,772 \\
(5,939-7,604)\end{array}$ & $\begin{array}{l}6,204 \\
(5,385-7,023)\end{array}$ & $\begin{array}{l}4,632 \\
(3,809-5,456)\end{array}$ & $\begin{array}{l}4,662 \\
(3,821-5,502)\end{array}$ & $<0.001$ & 0.762 & $<0.001$ \\
\hline 6-min walk distance, $\mathrm{m}^{\mathrm{b}}$ & $523(502-545)$ & $518(498-539)$ & $469(448-489)$ & $469(447-491)$ & $<0.001$ & 0.942 & $<0.001$ \\
\hline \multicolumn{8}{|l|}{ Dietary outcomes } \\
\hline DASH diet score ${ }^{a, b}$ & $5.4(4.9-5.8)$ & $3.4(3.0-3.8)$ & $5.8(5.4-6.2)$ & $3.6(3.2-4.1)$ & 0.109 & $<0.001$ & $<0.001$ \\
\hline Sodium, mg ${ }^{\mathbf{b}}$ & $\begin{array}{l}1867 \\
(1,640-2095)\end{array}$ & $\begin{array}{l}2,632 \\
(2,407-2,858)\end{array}$ & $\begin{array}{l}1769 \\
(1,550-1987)\end{array}$ & $\begin{array}{l}2,418 \\
(2,175-2,661)\end{array}$ & 0.189 & $<0.001$ & 0.002 \\
\hline Potassium, mg ${ }^{\mathbf{b}}$ & $\begin{array}{l}2,319 \\
(2098-2,540)\end{array}$ & $\begin{array}{l}1726 \\
(1,505-1947)\end{array}$ & $\begin{array}{l}2,609 \\
(2,396-2,821)\end{array}$ & $\begin{array}{l}1,645 \\
(1,410-1880)\end{array}$ & 0.372 & $<0.001$ & $<0.001$ \\
\hline Magnesium, mg ${ }^{\mathbf{b}}$ & $219(198-241)$ & 155 (134-177) & $245(224-266)$ & $153(130-176)$ & 0.291 & $<0.001$ & $<0.001$ \\
\hline Calcium, mg & $641(570-712)$ & $554(484-624)$ & $758(690-825)$ & $531(456-606)$ & 0.203 & $<0.001$ & 0.042 \\
\hline \multicolumn{8}{|l|}{ CVD risk factor outcomes } \\
\hline CVD risk rank ${ }^{a}$ & $60.2(56.3-64.1)$ & $\begin{array}{l}64.4 \\
(60.6-68.2)\end{array}$ & $\begin{array}{l}64.5 \\
(60.7-68.3)\end{array}$ & $\begin{array}{l}67.8 \\
(63.8-71.8)\end{array}$ & 0.056 & 0.058 & 0.009 \\
\hline Systolic BP, mm Hg & $128(125-131)$ & $131(128-134)$ & $129(126-132)$ & $129(126-132)$ & 0.868 & 0.209 & 0.654 \\
\hline Diastolic BP, mm Hg & $73.9(72.4-75.4)$ & $\begin{array}{l}76.2 \\
(74.7-77.6)\end{array}$ & $\begin{array}{l}75.7 \\
(74.2-77.1)\end{array}$ & $\begin{array}{l}75.2 \\
(73.7-76.8)\end{array}$ & 0.363 & 0.130 & 0.173 \\
\hline Total CVD meds DDD ${ }^{c}$ & $2.3(2.1-2.6)$ & $2.6(2.3-2.8)$ & $2.2(2.0-2.5)$ & $2.6(2.3-2.9)$ & 0.552 & 0.062 & 0.293 \\
\hline BP DDD ${ }^{b}$ & $1.5(1.3-1.8)$ & $1.8(1.5-2.0)$ & $1.3(1.0-1.5)$ & $1.9(1.6-2.2)$ & 0.883 & 0.003 & 0.081 \\
\hline Cholesterol DDD & $0.65(0.51-0.78)$ & $\begin{array}{l}0.70 \\
(0.57-0.83)\end{array}$ & $\begin{array}{l}0.77 \\
(0.64-0.90)\end{array}$ & $\begin{array}{l}0.59 \\
(0.45-0.73)\end{array}$ & 0.900 & 0.356 & 0.504 \\
\hline Diabetes DDD ${ }^{\mathbf{b}}$ & $0.18(0.14-0.23)$ & $\begin{array}{l}0.12 \\
(0.07-0.17)\end{array}$ & $\begin{array}{l}0.18 \\
(0.14-0.23)\end{array}$ & $\begin{array}{l}0.12 \\
(0.07-0.17)\end{array}$ & 0.340 & 0.026 & 0.052 \\
\hline Weight, $\mathbf{l b}^{\mathbf{b}}$ & $193.6(191-196)$ & $\begin{array}{l}196.9 \\
(195-199)\end{array}$ & $194(192-196)$ & $195(193-197)$ & 0.777 & 0.017 & 0.384 \\
\hline Body mass index, $\mathrm{kg} / \mathrm{m}^{2 \mathrm{~b}}$ & $31.7(31.3-32.1)$ & $\begin{array}{l}32.3 \\
(31.9-32.6)\end{array}$ & $\begin{array}{l}31.8 \\
(31.4-32.1)\end{array}$ & $\begin{array}{l}32.0 \\
(31.6-32.3)\end{array}$ & 0.872 & 0.023 & 0.360 \\
\hline Total cholesterol, mg/dL ${ }^{b}$ & $\begin{array}{l}183.1 \\
(175.7-190.4)\end{array}$ & $\begin{array}{l}191.1 \\
(184.1-198.1)\end{array}$ & $\begin{array}{l}181.5 \\
(174.5-188.6)\end{array}$ & $\begin{array}{l}190.5 \\
(183.1-197.9)\end{array}$ & 0.783 & 0.018 & 0.122 \\
\hline $\begin{array}{l}\text { Low-density lipoprotein } \\
\text { cholesterol, } \mathrm{mg} / \mathrm{dL}^{\mathrm{b}}\end{array}$ & $\begin{array}{l}104.3 \\
(98.2-110.4)\end{array}$ & $\begin{array}{l}108.6 \\
(102.8-114.5)\end{array}$ & $\begin{array}{l}103.3 \\
(97.4-109.2)\end{array}$ & $\begin{array}{l}110.9 \\
(104.7-117.0)\end{array}$ & 0.954 & 0.045 & 0.080 \\
\hline $\begin{array}{l}\text { High-density lipoprotein } \\
\text { cholesterol, } \mathrm{mg} / \mathrm{dL}^{\mathrm{b}}\end{array}$ & $55.4-(52.5-58.3)$ & $\begin{array}{l}60.2 \\
(57.4-63.0)\end{array}$ & $\begin{array}{l}54.7 \\
(52.0-57.5)\end{array}$ & $\begin{array}{l}56.1 \\
(53.2-59.1)\end{array}$ & 0.053 & 0.038 & 0.981 \\
\hline $\begin{array}{l}\text { Very low-density lipoprotein } \\
\text { cholesterol, mg/dL }\end{array}$ & $23.4(21.1-25.7)$ & $\begin{array}{l}22.2 \\
(20.1-24.4)\end{array}$ & $\begin{array}{l}23.5 \\
(21.4-25.7)\end{array}$ & $\begin{array}{l}23.4 \\
(21.1-25.7)\end{array}$ & 0.159 & 0.856 & 0.864 \\
\hline Insulin, ulU/mL ${ }^{b}$ & $12.0(10.0-14.0)$ & $\begin{array}{l}13.6 \\
(11.8-15.5)\end{array}$ & $\begin{array}{l}14.0 \\
(12.2-15.9)\end{array}$ & $\begin{array}{l}14.5 \\
(12.5-16.5)\end{array}$ & 0.044 & 0.190 & 0.094 \\
\hline Glucose, mmol/L & $\begin{array}{l}100.9 \\
(96.4-105.4)\end{array}$ & $\begin{array}{l}101.6 \\
(97.3-105.9)\end{array}$ & $\begin{array}{l}99.6 \\
(95.3-103.9)\end{array}$ & $\begin{array}{l}104.8 \\
(100.3-109.3)\end{array}$ & 0.242 & 0.107 & 0.187 \\
\hline
\end{tabular}


Table 2 Postintervention values adjusted for preintervention values of each respective variable, along with age, sex, race, history of CVD event, and pretreatment MoCA score (continued)

\begin{tabular}{|c|c|c|c|c|c|c|c|}
\hline \multirow[b]{3}{*}{ Variable } & \multicolumn{4}{|c|}{ Individual intervention groups (by exercise factor) } & \multirow[b]{3}{*}{$\begin{array}{l}\text { Exercise } \\
\text { factor }\end{array}$} & \multirow[b]{3}{*}{$\begin{array}{l}\text { DASH } \\
\text { factor }\end{array}$} & \multirow[b]{3}{*}{$\begin{array}{l}\text { Combo } \\
\text { vs HE }\end{array}$} \\
\hline & Exercise & & No exercis & & & & \\
\hline & $\begin{array}{l}\text { DASH } \\
(\text { AE + DASH }) \\
(n=40)\end{array}$ & $\begin{array}{l}\text { No-DASH } \\
\text { (AE) }(n=41)\end{array}$ & $\begin{array}{l}\text { DASH } \\
\text { (DASH) } \\
(\mathrm{n}=41)\end{array}$ & $\begin{array}{l}\text { No-DASH } \\
(\mathrm{HE})(\mathrm{n}=38)\end{array}$ & & & \\
\hline Triglycerides, mg/dL & $116.6(105-128)$ & $\begin{array}{l}111.2 \\
(101-122)\end{array}$ & $\begin{array}{l}117.8 \\
(107-129)\end{array}$ & $\begin{array}{l}117.2 \\
(106-129)\end{array}$ & 0.146 & 0.873 & 0.824 \\
\hline
\end{tabular}

Abbreviations: $\mathrm{AE}=$ aerobic exercise; $\mathrm{BP}=$ blood pressure; CVD = cardiovascular disease; DASH = Dietary Approaches to Stop Hypertension; DDD = daily defined dose; $\mathrm{ETT}$ = exercise tolerance test; $\mathrm{HE}$ = health education; MoCA = Montreal Cognitive Assessment.

a The principal outcome variable selected a priori within each domain.

${ }^{\mathrm{b}} p<0.05$ for treatment changes on at least 1 factor (uncorrected).

medication load by one-quarter daily dose $(-0.24[-0.06$ to $-0.42]$ DDD, $p=0.003)$. Participants in the exercise factor groups ( $\mathrm{AE}+\mathrm{DASH}$ and $\mathrm{AE}$ alone) exhibited greater improvements in insulin compared to participants in the nonexercise conditions $(-1.16[-2.5$ to 0.2$], p=0.044)$.

\section{Intervention effects on neurocognitive function}

Participants in the $\mathrm{AE}$ conditions exhibited significant improvements in executive function $(\mathrm{b}=4.2[0.2-8.2], \mathrm{d}=$ $0.32, p=0.046$ ), whereas no significant changes in executive function were observed for those in the DASH diet condition $(\mathrm{b}=3.7[-0.2$ to 7.7$], \mathrm{d}=0.30, p=0.059)$ (figure $2 \mathrm{~A}$ ). Explanatory follow-up testing revealed that the largest improvements were achieved by participants in $\mathrm{AE}+\mathrm{DASH}$ $(\mathrm{d}=0.40, p=0.012)$ compared to the HE controls. To illustrate the potential clinical significance of these changes, we estimated changes in predicted age based on the participants' performance on the executive function subtests for which published estimates were available (i.e., TMT-A, TMTB, and Stroop color-word). At baseline, participants exhibited performance consistent with individuals in their early 90 s (mean 93.3 [SD 45] years), $\approx 28$ years older than their chronologic age. After 6 months, the performance of individuals in the $\mathrm{AE}+\mathrm{DASH}$ condition was consistent with younger predicted age, with improvements corresponding to an -8.8 - (-18.7 to 1.0$)$ year improvement. In contrast, the estimated performance of participants in the HE control group was $\approx 6$ months worse than their baseline, which actually was the duration of the intervention, demonstrating an estimated $0.5-(-9.2$ to 10.3$)$ year worsening performance.

For the analysis of global cognitive functioning, significant improvements were found for the exercise factor on the

Figure 2 Intervention effects for (A) global executive function and (B) mCDR

A. Global executive functioning

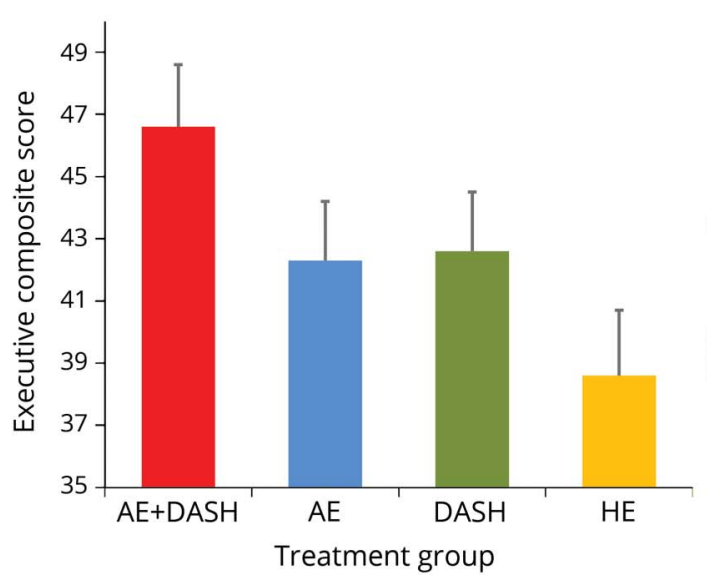

B. Clinical dementia rating

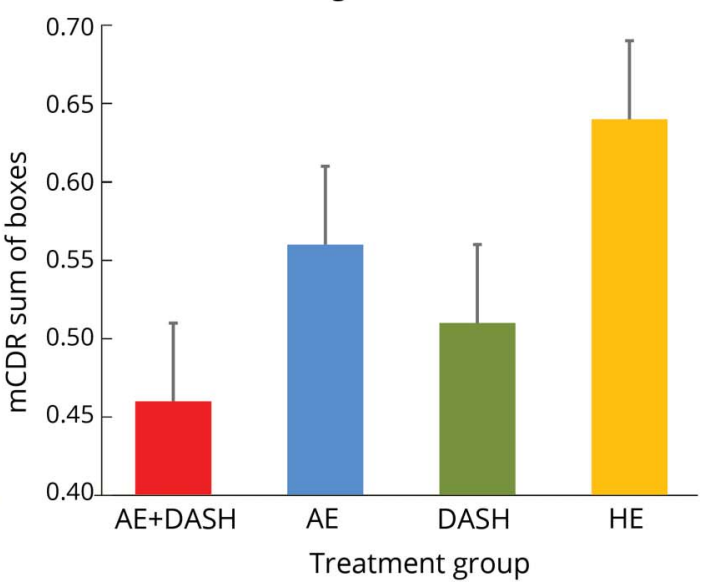

Values for the global composite are presented as mean postintervention ranks adjusted for preintervention rank, age, education, sex, ethnicity, baseline Montreal Cognitive Assessment (MoCA) score, APOE genotype, Framingham Stroke Risk Profile (FSRP), and anti-inflammatory and cardiovascular disease (CVD) medications. Higher scores represent better performance. For the modified Clinical Dementia Rating (mCDR), values are presented as mean postintervention mCDR Sum of Boxes scores adjusted for preintervention scores, age, education, sex, ethnicity, pretreatment MoCA score, APOE genotype, FSRP, anti-inflammatory, and CVD medications. Lower scores represent greater improvement. AE = aerobic exercise; DASH = Dietary Approaches to Stop Hypertension; $\mathrm{HE}=$ health education 
modified CDR Sum of Boxes $(d=0.36, p=0.027)$; the combined exercise and DASH diet group had significantly lower scores compared to HE controls $(\mathrm{d}=0.39, p=0.007)$ (figure 2B). The DASH factor was not significant, however $(\mathrm{d}=0.19, p=0.244)$. No intervention-related improvements were observed for the memory domain (exercise factor: $\mathrm{d}=$ $0.19, p=0.236$; DASH factor: $\mathrm{d}=0.15, p=0.351$ ) or the language/verbal fluency domain (exercise factor: $\mathrm{d}=0.12, p=$ 0.449; DASH factor: $\mathrm{d}=0.23, p=0.241$ ), and no participants converted to dementia.

\section{Correlates of neurocognitive change}

To explore potential mechanisms by which the interventions affected neurocognition, we examined the associations between improvements in peak $\mathrm{VO}_{2}$, our composite CVD risk marker, and components of the DASH diet with changes in neurocognition. Examination of intervention changes revealed that improvements in aerobic fitness $\left(\mathrm{VO}_{2}\right.$ peak; $\mathrm{b}=2.3, p<$ $0.049)$ and reduced CVD risk $(b=2.6, p<0.042)$ were associated with improvements in executive function. Examination of DASH diet nutritional components revealed that reduced sodium intake was associated with improved executive function $(b=0.18, p=0.024)$, whereas changes in potassium $(p=0.955)$, magnesium $(p=0.820)$, and calcium $(p=0.663)$ intake were not related to executive function.

\section{Discussion}

Results of this randomized clinical trial demonstrate that a 6-month program of $\mathrm{AE}$ resulted in significant improvements in executive functioning in older adults with CVD risk factors, subjective cognitive complaints, and CIND. Participants who engaged in regular AE 3 times per week for 6 months demonstrated improved performance on a standard battery of neurocognitive tests of executive function. Our findings are strengthened by our high level of internal validity, with no dropouts, a comprehensive neurocognitive test battery using blinded assessors, a substantial proportion of minority participants, and objective improvements in physical activity, aerobic capacity, and adherence to the DASH eating plan. The DASH factor was not related to improved neurocognition, although we noted that greater benefits were observed among those participants who engaged in both exercise and the DASH eating plan compared to HE controls. While this overall pattern of results may suggest a potential additive benefit of AE and the DASH diet, we caution that our sample was small, and these findings must be interpreted with caution.

To the best of our knowledge, ENLIGHTEN may be the first randomized trial to examine the independent and combined effects of a whole diet and $\mathrm{AE}$ program on neurocognitive function in older adults at heightened risk for progressive decline in cognitive functioning. In a previous study using a 2-by-2 design, 152 participants with mild cognitive impairment were randomized to 1 year of moderate-intensity walking or placebo activity twice a week and vitamin B supplementation or placebo for 1 year. Neither intervention improved cognition, however. ${ }^{32}$ In contrast, previous observational studies have shown that increased physical activity was associated with lower risk of cognitive decline in healthy older adults. ${ }^{33}$ However, results of meta-analyses of randomized trials have been inconsistent, with some studies reporting significant benefit, ${ }^{34,35}$ and others reporting no benefit compared to nonexercising controls. ${ }^{36}$

One important source of variation that likely contributes to inconsistencies across studies concerns the selection of neurocognitive tests, with no standard test battery used universally. It has been suggested that the benefits of exercise may be specific to tasks of executive control functions but not to tasks of memory or nonexecutive functions. ${ }^{18}$ While our results provide support for the notion that exercise may be especially beneficial for executive control functions, it is possible that the pattern of findings may have resulted from our preferential inclusion of individuals with frontal lobe deficits and vascular CIND, a clinical condition predominated by impairments in executive function and processing speed. ${ }^{21}$

Previous observational studies have found that greater intake of fruits, vegetables, and low-fat dairy products is associated not only with improved CVD health but also with improved cognition and lower risk of cognitive decline, ${ }^{15,37}$ dementia, ${ }^{38}$ and Alzheimer disease. ${ }^{39}$ There have been few whole-diet interventions, however. The Mediterranean diet, supplemented with olive oil, has been shown to produce better cognitive performance compared to low-dietary-fat controls. ${ }^{40}$ The DASH eating plan is similar in its emphasis on fruits, vegetables, and whole grains but places greater emphasis on low-fat dairy products, as well as increased potassium and reduced sodium intake. Data from randomized trials have confirmed the benefits of the DASH diet in lowering blood pressure, ${ }^{41}$ improving vascular biomarkers, ${ }^{17}$ and enhancing cognitive performance, particularly when combined with weight reduction. ${ }^{19}$ Although there is evidence that weight loss also could be beneficial, ${ }^{42,43}$ the DASH intervention used in the ENLIGHTEN study was not designed to promote weight loss, and there was no differential weight loss between the DASH and non-DASH intervention groups. Moreover, we did not find that the DASH diet alone was associated with improved neurocognition in this study.

Growing evidence supports the value of multicomponent interventions in mitigating cognitive decline. In an observational study, Scarmeas and colleagues ${ }^{13}$ showed that adherence to the Mediterranean-type diet and higher physical activity were associated with reduced risk for dementia, while the Finnish Geriatric Intervention Study to Prevent Cognitive Impairment and Disability (FINGER) randomized trial demonstrated that an intensive, 2-year, multicomponent intervention that included $\mathrm{AE}$, vascular risk management, dietary modification, and cognitive training was associated with improved cognitive performance among older adults at risk for dementia. ${ }^{44}$ Similarly, recent meta-analytic reviews have 
suggested that exercise programs that are supplemented by additional interventions confer larger cognitive benefits compared to interventions using exercise alone. ${ }^{45}$

Although the mechanisms responsible for their benefits are not known, there are a number of plausible biological pathways by which lifestyle factors may affect neurocognition. Vascular abnormalities, including hypertension, diabetes mellitus, and dyslipidemia, have been associated with cognitive impairments and dementia ${ }^{46}$ and may be improved by diet and exercise. Nonvascular mechanisms are also possible, including improved modulation of growth factor expression, reduced oxidative stress, improved cerebrovascular functioning, reduced neuroinflammation, and enhanced insulindependent energy metabolism. ${ }^{47}$ Reduction of sodium intake also is associated with improved blood-brain barrier integrity ${ }^{48,49}$ In the ENLIGHTEN study, a post hoc correlational analysis revealed that improved aerobic fitness, reduced CVD risk, and reductions in dietary sodium were all independently predictive of improved executive function. This pattern of exploratory results suggests the need for further research examining whether improvements in neurocognition may be best achieved through multiple interventional approaches and their overlapping benefits and mechanistic pathways.

\section{Limitations}

Our sample consisted of sedentary adults with CVD risk factors and subjective memory or other cognitive concerns with mild, preclinical cognitive impairments. Examination of demographically corrected performance suggested that only $25 \%$ of participants fell below the normal range on delayed recall memory measures at baseline, whereas $45 \%$ fell below the normal range on tests of executive function. Participants were highly motivated; we did not have a single dropout. Although the results may not generalize to less motivated groups, our findings demonstrate that high levels of adherence to lifestyle modifications can be achieved in older adults even with cognitive impairments. The present trial was only 6 months in duration; therefore, we are unable to ascertain the longer-term effects of lifestyle modification on cognitive outcomes. Given the beneficial effects observed on measures of executive function and the CDR interview ratings, our results suggest that the observed benefit for executive functioning may translate into improved functional outcomes even within 6 months. The ENLIGHTEN trial was conducted at a single site and was relatively small $(\mathrm{n}=160)$ compared with recent multicenter trials. ${ }^{44}$ Our study may have been underpowered to detect differences between exercise and the DASH diet alone and therefore provides limited evidence of the relative benefits of these 2 interventions. In addition, our findings may have resulted from our preferential inclusion of individuals with frontal lobe deficits and vascular CIND and because our assessment of language/verbal fluency was limited to only the COWA and Animal Naming tests, which may not have been sufficient to adequately assess this cognitive domain. Mechanisms for benefit also remain uncertain because we did not collect neuroimaging or other CNS measures of cerebrovascular changes, which could have provided greater insight into possible mechanisms responsible for the observed improvements in executive functioning.

\section{Author contributions}

James A. Blumenthal, $\mathrm{PhD}$ : study concept and design, analysis and interpretation, manuscript preparation, study supervision. Patrick J. Smith, PhD: acquisition of data, analysis and interpretation, critical revision of the manuscript for important intellectual content, statistical analysis, study supervision. Stephanie Mabe, MS: acquisition of data, critical revision of the manuscript for important intellectual content, study supervision. Alan Hinderliter, MD: study concept and design, analysis and interpretation, critical revision of the manuscript for important intellectual content. Pao-Hwa Lin, $\mathrm{PhD}$ : study concept and design, acquisition of data, analysis and interpretation, critical revision of the manuscript for important intellectual content, study supervision. Lawrence Liao, MD: acquisition of data, critical revision of the manuscript for important intellectual content. Kathleen A. Welsh-Bohmer, PhD: study concept and design, critical revision of the manuscript for important intellectual content. Jeffrey N. Browndyke, $\mathrm{PhD}$ : study concept and design, critical revision of the manuscript for important intellectual content, study supervision. William E. Kraus, MD: acquisition of data, critical revision of the manuscript for important intellectual content, study supervision. P. Murali Doraiswamy, MD, and James R. Burke $\mathrm{MD}, \mathrm{PhD}$ : study concept and design, critical revision of the manuscript for important intellectual content. Andrew Sherwood, PhD: study concept and design, acquisition of data, analysis and interpretation, critical revision of the manuscript for important intellectual content, study supervision.

\section{Acknowledgment}

The authors thank the members of their Data and Safety Monitoring Board: Diane Catellier, $\mathrm{PhD}$ (chair), David Sheps, $\mathrm{MD}$, and Robert Carney, $\mathrm{PhD}$, for their guidance and oversight of this study. Thanks also are extended to Cassandra Germain, PhD, Jeanne Schwartz, PA-C, Michael Ellis, BS, Carola Ekelund, PT, Megan Reaves, MS, Kenlyn Young, RD, and Julie Johnson, PA-C, and to research assistants Molly McLaren, Kathryn Sommers, Natalie Allcott, Brittany Manobianco, Brendan Wesp, Megan Gurjar, Alexa Waters, and Amelia Hoyle. The authors also thank the staff at the respective exercise sites under the direction of Karen Craig (Duke), Paula Miller, MD (University of North Carolina), and Andrea Layton (Duke Raleigh) for their support.

\section{Study funding}

The study was supported by a grant from the NIH (HL109219). All coauthors received some salary support for their effort on the project.

\section{Disclosure}

The authors report no disclosures relevant to the manuscript. Go to Neurology.org/N for full disclosures. 


\section{Publication history}

Received by Neurology May 19, 2018. Accepted in final form September $18,2018$.

\section{References}

1. Plassman BL, Langa KM, Fisher GG, et al. Prevalence of cognitive impairment without dementia in the United States. Ann Intern Med 2008;148:427-434.

2. Graham JE, Rockwood K, Beattie BL, et al. Prevalence and severity of cognitive impairmen with and without dementia in an elderly population. Lancet 1997;349:1793-1796.

3. Haan MN, Wallace R. Can dementia be prevented? Brain aging in a population-based context. Annu Rev Public Health 2004;25:1-24.

4. Luchsinger JA. Adiposity, hyperinsulinemia, diabetes and Alzheimer's disease: an epidemiological perspective. Eur J Pharmacol 2008;585:119-129.

5. Yaffe K. Metabolic syndrome and cognitive disorders: is the sum greater than its parts? Alzheimer Dis Assoc Disord 2007;21:167-171.

6. Alzheimer's Association. Alzheimer's facts and figures [online]. Available at: alz.org/ alzheimers_disease_facts_and_figures.asp. Accessed July 31, 2018

7. Wen CP, Wai JP, Tsai MK, et al. Minimum amount of physical activity for reduced mortality and extended life expectancy: a prospective cohort study. Lancet 2011;378: $1244-1253$.

8. Larson EB, Wang L, Bowen JD, et al. Exercise is associated with reduced risk for incident dementia among persons 65 years of age and older. Ann Intern Med 2006;144:73-81.

9. Rovio S, Kåreholt I, Helkala EL, et al. Leisure-time physical activity at midlife and the risk of dementia and Alzheimer's disease. Lancet Neurol 2005;4:705-711.

10. Angevaren M, Aufdemkampe G, Verhaar HJ, Aleman A, Vanhees L. Physical activity and enhanced fitness to improve cognitive function in older people without known cognitive impairment. Cochrane Database Syst Rev 2008:CD005381.

11. Heyn P, Abreu BC, Ottenbacher KJ. The effects of exercise training on elderly persons with cognitive impairment and dementia: a meta-analysis. Arch Phys Med Rehabil 2004;85:1694-1704.

12. Barberger-Gateau P, Raffaitin C, Letenneur L, et al. Dietary patterns and risk of dementia: the Three-City Cohort Study. Neurology 2007;69:1921-1930.

13. Scarmeas N, Luchsinger JA, Schupf N, et al. Physical activity, diet, and risk of Alzheimer disease. JAMA 2009;302:627-637.

14. Scarmeas N, Stern Y, Tang MX, Mayeux R, Luchsinger JA. Mediterranean diet and risk for Alzheimer's disease. Ann Neurol 2006;59:912-921.

15. Wengreen H, Munger RG, Cutler A, et al. Prospective study of Dietary Approaches to Stop Hypertension- and Mediterranean-style dietary patterns and age-related cognitive change: the Cache County Study on Memory, Health and Aging. Am J Clin Nutr 2013;98:1263-1271.

16. Chobanian AV, Bakris GL, Black HR, et al. Seventh report of the Joint National Committee on Prevention, Detection, Evaluation, and Treatment of High Blood Pressure. Hypertension 2003;42:1206-1252.

17. Blumenthal JA, Babyak MA, Sherwood A, et al. Effects of the Dietary Approaches to Stop Hypertension diet alone and in combination with exercise and caloric restriction on insulin sensitivity and lipids. Hypertension 2010;55:1199-1205.

18. Kramer AF, Hahn S, Cohen NJ, et al. Ageing, fitness and neurocognitive function. Nature 1999;400:418-419.

19. Smith PJ, Blumenthal JA, Babyak MA, et al. Effects of the Dietary Approaches to Stop Hypertension diet, exercise, and caloric restriction on neurocognition in overweight adults with high blood pressure. Hypertension 2010;55:1331-1338.

20. Colcombe S, Kramer AF. Fitness effects on the cognitive function of older adults: a meta-analytic study. Psychol Sci 2003;14:125-130.

21. Hachinski V, Iadecola C, Petersen RC, et al. National Institute of Neurological Disorders and Stroke-Canadian Stroke Network vascular cognitive impairment harmonization standards. Stroke 2006;37:2220-2241.

22. Knopman DS, Weintraub S, Pankratz VS. Language and behavior domains enhance the value of the clinical dementia rating scale. Alzheimers Dement 2011;7:293-299.

23. Blumenthal JA, Rejeski WJ, Walsh-Riddle M, et al. Comparison of high- and lowintensity exercise training early after acute myocardial infarction. Am J Cardiol 1988; 61:26-30.

24. Epstein DE, Sherwood A, Smith PJ, et al. Determinants and consequences of adherence to the Dietary Approaches to Stop Hypertension diet in African-American and white adults with high blood pressure: results from the ENCORE trial. J Acad Nutr Diet 2012;112:1763-1773.
25. Folsom AR, Parker ED, Harnack LJ. Degree of concordance with DASH die guidelines and incidence of hypertension and fatal cardiovascular disease. Am J Hypertens 2007;20:225-232.

26. WHO Collaborating Centre for Drug Statistics Methodology. Daily defined dose Definition and general considerations [online]. Available at http://www.whocc.no/ ddd/definition and general considera/.

27. D'Agostino RB, Wolf PA, Belanger AJ, Kannel WB. Stroke risk profile: adjustment for antihypertensive medication: the Framingham Study. Stroke 1994;25:40-43.

28. Koch GG, Gansky SA. Statistical considerations for multiplicity in confirmatory protocols. Drug Inf J 1996;30:523-533.

29. Felker GM, Maisel AS. A global rank end point for clinical trials in acute heart failure. Circ Heart Fail 2010;3:643-646.

30. Lehmacher W, Wassmer G, Reitmeir P. Procedures for two-sample comparisons with multiple endpoints controlling the experimentwise error rate. Biometrics 1991;47:511-521.

31. Attix DK, Story TJ, Chelune GJ, et al. The prediction of change: normative neuropsychological trajectories. Clin Neuropsychol 2009;23:21-38.

32. van Uffelen JG, Chinapaw MJ, van MW, Hopman-Rock M. Walking or vitamin B for cognition in older adults with mild cognitive impairment? A randomised controlled trial. Br J Sports Med 2008;42:344-351.

33. Hamer M, Chida Y. Physical activity and risk of neurodegenerative disease: a systematic review of prospective evidence. Psychol Med 2009;39:3-11.

34. Smith PJ, Blumenthal JA, Hoffman BM, et al. Aerobic exercise and neurocognitive performance: a meta-analytic review of randomized controlled trials. Psychosom Med 2010;72:239-252.

35. Zheng G, Xia R, Zhou W, Tao J, Chen L. Aerobic exercise ameliorates cognitive function in older adults with mild cognitive impairment: a systematic review and meta-analysis of randomised controlled trials. Br J Sports Med 2016;50:1443-1450.

36. Young J, Angevaren M, Rusted J, Tabet N. Aerobic exercise to improve cognitive function in older people without known cognitive impairment. Cochrane Database Syst Rev 2015:CD005381.

37. Del Parigi A, Panza F, Capurso C, Solfrizzi V. Nutritional factors, cognitive decline, and dementia. Brain Res Bull 2006;69:1-19.

38. Luchsinger JA, Mayeux R. Dietary factors and Alzheimer's disease. Lancet Neurol 2004;3:579-587.

39. Essink-Bot ML, Pereira J, Packer C, Schwarzinger M, Burstrom K. Cross-national comparability of burden of disease estimates: the European Disability Weights Project. Bull World Health Organ 2002;80:644-652.

40. Martinez-Lapiscina EH, Clavero P, Toledo E, et al. Mediterranean diet improves cognition: the PREDIMED-NAVARRA randomised trial. J Neurol Neurosurg Psychiatry 2013;84:1318-1325.

41. Appel LJ, Champagne CM, Harsha DW, et al. Effects of comprehensive lifestyle modification on blood pressure control: main results of the PREMIER clinical trial. JAMA 2003;289:2083-2093.

42. Siervo M, Arnold R, Wells JC, et al. Intentional weight loss in overweight and obese individuals and cognitive function: a systematic review and meta-analysis. Obes Rev 2011;12:968-983.

43. Witte AV, Fobker M, Gellner R, Knecht S, Flöel A. Caloric restriction improves memory in elderly humans. Proc Natl Acad Sci USA 2009;106:1255-1260.

44. Ngandu T, Lehtisalo J, Solomon A, et al. A 2 year multidomain intervention of diet, exercise, cognitive training, and vascular risk monitoring versus control to preven cognitive decline in at-risk elderly people (FINGER): a randomised controlled trial. Lancet 2015;385:2255-2263.

45. Sáez de Asteasu ML, Martínez-Velilla N, Zambom-Ferraresi F, Casas-Herrero Á, Izquierdo $\mathrm{M}$. Role of physical exercise on cognitive function in healthy older adults: a systematic review of randomized clinical trials. Ageing Res Rev 2017;37:117-134

46. Akinyemi RO, Mukaetova-Ladinska EB, Attems J, Ihara M, Kalaria RN. Vascular risk factors and neurodegeneration in ageing related dementias: Alzheimer's disease and vascular dementia. Curr Alzheimer Res 2013;10:642-653.

47. Greenwood PM, Parasuraman R. Neuronal and cognitive plasticity: a neurocognitive framework for ameliorating cognitive aging. Front Aging Neurosci 2010;2:150

48. Fiocco AJ, Shatenstein B, Ferland G, et al. Sodium intake and physical activity impact cognitive maintenance in older adults: the NuAge Study. Neurobiol Aging 2012;33: 829.e821-e828.

49. Heye AK, Thrippleton MJ, Chappell FM, et al. Blood pressure and sodium: association with MRI markers in cerebral small vessel disease. J Cereb Blood Flow Metab 2016;36:264-274 


\section{Neurology}

\section{Lifestyle and neurocognition in older adults with cognitive impairments: A randomized trial}

James A. Blumenthal, Patrick J. Smith, Stephanie Mabe, et al.

Neurology 2019;92;e212-e223 Published Online before print December 19, 2018

DOI 10.1212/WNL.0000000000006784

This information is current as of December 19, 2018

\section{Updated Information \&} Services

References

Citations

Subspecialty Collections

Permissions \& Licensing

Reprints including high resolution figures, can be found at: http://n.neurology.org/content/92/3/e212.full

This article cites 45 articles, 10 of which you can access for free at: http://n.neurology.org/content/92/3/e212.full\#ref-list-1

This article has been cited by 1 HighWire-hosted articles: http://n.neurology.org/content/92/3/e212.full\#\#otherarticles

This article, along with others on similar topics, appears in the following collection(s):

Clinical trials Randomized controlled (CONSORT agreement)

http://n.neurology.org/cgi/collection/clinical_trials_randomized_contro

lled_consort_agreement

Cognitive aging

http://n.neurology.org/cgi/collection/cognitive_aging

Executive function

http://n.neurology.org/cgi/collection/executive_function

Information about reproducing this article in parts (figures,tables) or in its entirety can be found online at:

http://www.neurology.org/about/about_the_journal\#permissions

Information about ordering reprints can be found online:

http://n.neurology.org/subscribers/advertise

Neurology ${ }^{\circledR}$ is the official journal of the American Academy of Neurology. Published continuously since 1951, it is now a weekly with 48 issues per year. Copyright () 2018 American Academy of Neurology. All rights reserved. Print ISSN: 0028-3878. Online ISSN: 1526-632X.

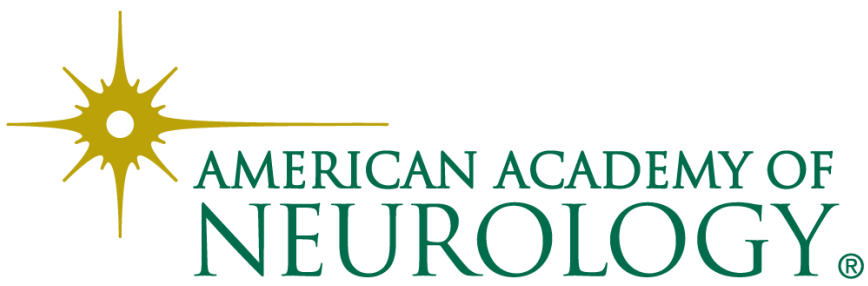

